\title{
Biomechanical modelling of colorectal crypt budding and fission
}

\author{
Carina M. Edwards · S. Jonathan Chapman
}

Received: date / Accepted: date

\begin{abstract}
This paper presents a biomechanical model for the small pits, called crypts, that line the colon. A continuum approach is adopted, with the crypt epithelium modelled as a growing beam attached to the underlying lamina by cell bonds, which generate tension within the layer. These cell attachments are assumed to be viscoelastic thus allowing for cell progression along the crypt. It is shown that any combination of: an increase in net proliferation (i.e. cell production minus apoptosis), an enlargement of the proliferative compartment, an increase in the strength of the cellular attachment to the underlying lamina, or a change in the rate of cell growth or cell bonding may generate buckling of the tissue. These changes can all be generated by an activating mutation of the Wnt cascade, which is generally accepted to be the first genetic change in colorectal cancer, with subsequent deformation, budding, and crypt fission an observed feature of the adenomatous crypt.
\end{abstract}

Keywords Morphogenesis · Buckling $\cdot$ Colorectal Cancer $\cdot$ Beam · Elasticity $\cdot$ Growth

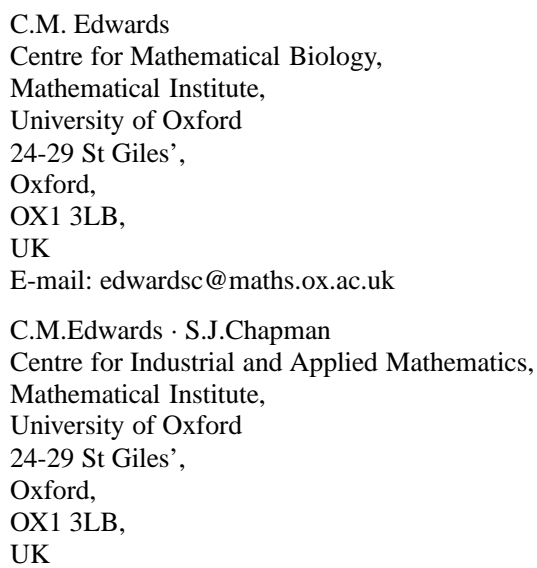




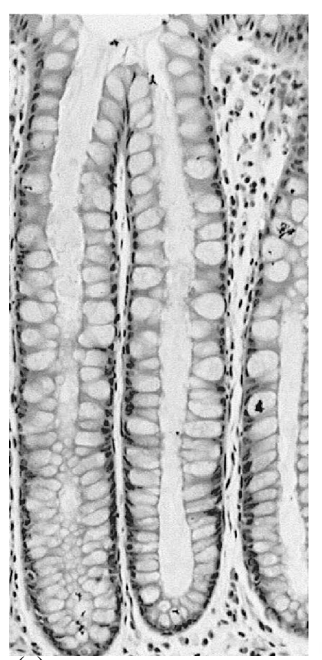

(a)

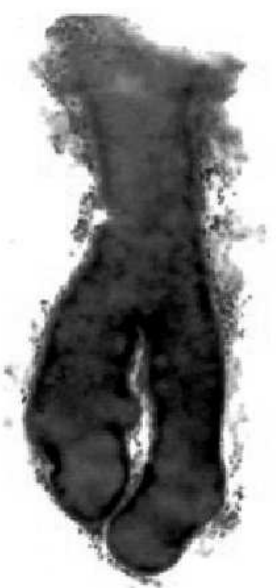

(b)

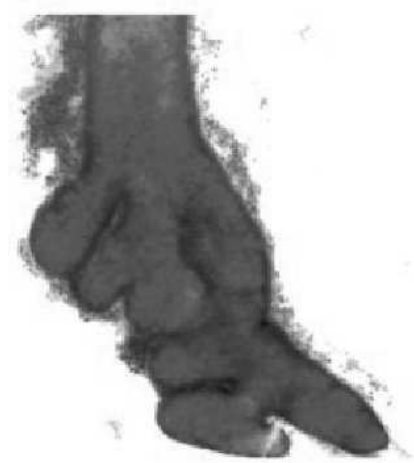

Fig. 1 (a) A section through a normal healthy crypt. Note the smooth straight sides and regular shape. (Image reproduced from (Shih et al., 2001). Copyright 2001 National Academy of Sciences U.S.A.). (b) Colorectal crypt budding and fission in an adenoma. (Image reproduced with permission from (Preston et al., 2003)).

\section{Introduction}

Colorectal cancer is initiated in the small pits, called crypts, that line the surface of the colon. A section of a healthy crypt is shown in Figure 1(a). The crypt is lined with epithelial cells, which are attached to the basal lamina, which itself lies on top of the lamina propria, see Figure 2. At the bottom of each crypt lie stem cells which produce semi-differentiated transit cells that proliferate and move up the crypt. As these transit cells progress along the crypt they differentiate, stop dividing and are eventually shed into the lumen of the colon; the turnover of cells in the crypt is rapid, of the order of days (Marshman et al., 2002).

It is generally accepted that one of the earliest genetic events that leads to cancer developing is an activating mutation in the Wnt cascade, normally the loss of the APC gene (Reya and Clevers, 2005). The main effect of this change is to generate hyperproliferation in the crypt, although the Wnt pathway and APC are also implicated in the control of cell-cell and cell-matrix adhesion, motility and cell death (Fodde, 2003; Nelson and Nusse, 2004; Samsom et al., 2004). Following this initial mutational change in the crypt the development of the tumour follows a multi-step sequence whereby further genetic alterations usually lead to the development of a polyp-like adenoma, which subsequently develops into a cancerous lesion. This progression of the disease is known as the adenoma-carcinoma sequence (Fearon and Vogelstein, 1990).

Within adenomas and hyperplastic polyps it is observed that crypts are elongated and deformed, exhibiting budding and multiple fission events (Araki et al., 1995; Brittan and Wright, 2004; Wong et al., 2002). (Although crypt fission is a normal process in the human colon the rate of crypt division in adenomas is significantly raised). Examples of these deformations are shown in Figure 1(b). These observations have led to the suggestion that adenomatous regions initially grow by crypt fission (Greaves et al., 2006; Preston et al., 2003; Renehan et al., 2002; Roncucci et al., 2000), although another mechanism for ade- 


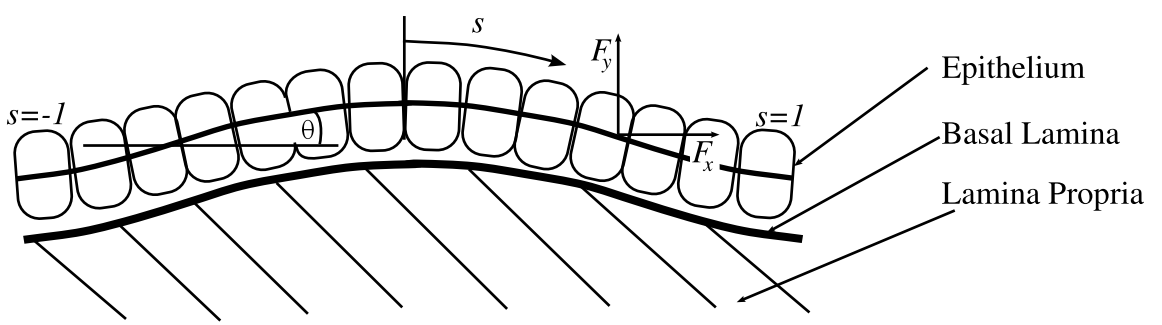

Fig. 2 A schematic representation of the 'unfolded' crypt in a buckled configuration. The epithelium is modelled as a beam. The arc length $s$ is measured from the centre of the crypt, $\theta$ is the angle the beam makes with the horizontal, and $F_{x}, F_{y}$ are forces generated within the beam in the $x$ and $y$ directions respectively.

noma growth is the invasion of dysplastic cells into neighbouring crypts from the 'top-down' (Shih et al., 2001).

The aim of this paper is to show how an activating mutation in the Wnt cascade could biomechanically initiate crypt budding and fission, and to predict when these events will occur.

Previous biomechanical models for the folding of epithelial layers have mainly focused on apical contractions of the epithelial cells, whereby the cells change their shape from rectangular to trapezoidal thus changing the local layer curvature, in response to a chemical or mechanical stimulus (e.g. Odell et al., 1981; Cummings, 1990). This modelling approach has been used to describe, for example, gastrulation (Keller et al., 2003), and sea urchin invagination (Davidson et al., 1995). However, for colorectal crypt budding and fission to be initiated by changes in the Wnt cascade the folding must be driven by hyperproliferation, or by changes in cell motility and adhesion, and not by such apical contractions.

A previous model of colorectal crypt fission by Drasdo and Loeffler (2001) used an off-lattice cellular automata model to look at the effect of cell growth on the structure of the crypt. The crypt was modelled as a u-shaped chain of deformable, growing circles. The destabilising effect of this growth was countered by the bending stiffness of the connections between the cells and a stabilising elastic force due to the surrounding tissue. It was shown that by reducing the bending stiffness of the connections between cells and the cell cycle times a buckled arrangement of cells could be generated through the destabilising effect of the growth. This result was then compared with a simple continuum calculation where the growth of the cell chain is stabilised by bending stiffness alone.

In this paper, we present a model of colorectal crypt budding and fission that will allow us to determine quantitatively how the crypt shape is affected by any combination of the possible effects of an activating mutation in the Wnt cascade. We adopt a continuum modelling approach and consider the crypt to be a continuous layer of tissue. For simplicity, we will consider a two-dimensional section and 'unfold' the crypt, modelling the epithelial layer as a beam attached to an underlying mucosa. It is these cell attachments that generate the stress within the beam as it grows, as well as stabilising the beam in the perpendicular direction. To allow for the dynamic detachment and reattachment of the cells as they move along the crypt we model the cell attachments by viscoelastic springs.

We will present this continuum model for the crypt in Section 3. However, we first in Section 2 consider the paradigm problem of a beam attached to an elastic foundation, which is approximated by linear springs, buckled by an applied force (a problem occasionally referred to as a Winkler column). The linearisation of this simple problem is considered in, for example, (Brush and Almroth, 1975) for a simply supported beam. Variations on the 
model include considering that the beam is under load rather than compression (Timoshenko and Lessells, 1928), or that the springs are nonlinear in response (Hunt et al., 1989). We will then extend this model to include the extensibility of the tissue.

\section{A paradigm problem}

We consider that the crypt's normal steady state configuration is that of a flat tissue layer. We model this thin layer as a beam whose shape is given by the nondimensional beam equation

$$
\frac{\mathrm{d}^{2} \theta}{\mathrm{d} s^{2}}+\lambda_{0}\left(F_{y} \cos \theta-F_{x} \sin \theta\right)=0,
$$

where $\theta$ is the angle the beam makes with the horizontal, $s$ is the arc length along the beam measured from its centre, and $F_{x}$ and $F_{y}$ are the forces generated within the beam in the $x$ and $y$ directions respectively, as illustrated in Figure 2.

We model the cellular attachment to the underlying mucosa as springs, in which case the forces $F_{x, y}$ may be determined from a simple force balance as

$$
\begin{aligned}
& \frac{\mathrm{d} F_{x}}{\mathrm{~d} s}=T_{x}, \\
& \frac{\mathrm{d} F_{y}}{\mathrm{~d} s}=T_{y},
\end{aligned}
$$

where $T_{x, y}$ is the tension generated within the spring in the $x$ and $y$ directions respectively. We begin by considering a simple paradigm problem in which the springs are taken as being purely elastic, so that

$$
\begin{aligned}
& T_{x}=\int_{0}^{s} \cos \theta \mathrm{d} s-f(s), \\
& T_{y}=\int_{-1}^{s} \sin \theta \mathrm{d} s,
\end{aligned}
$$

where $f(s)$ maps the deformed configuration of the beam back to the initial flat configuration $(X, Y)=(f(s), 0)$. The variables have been made nondimensional by scaling the arc length $s$ with the crypt depth $L$, and $F_{x}$ and $F_{y}$ with $k L^{2}$ where $k$ is the spring constant of the attachments. The nondimensional parameter $\lambda_{0}=k L^{4} / B$, where $B$ is the bending stiffness of the tissue layer.

We initially consider a simple paradigm problem in which the buckling of the layer is driven by an externally applied force, and so apply the boundary conditions

$$
F_{x}=-F_{0}, \quad F_{y}=0, \quad \theta=0, \quad \text { at } s= \pm 1,
$$

where we have assumed that the beam is clamped at the ends. Although the applied force $F_{0}$ is not biologically realistic, this simple problem will give us insight into how the system will behave when stress is generated in the epithelial layer as a result of growth. In the next section, when we introduce tissue growth in the model, we will apply zero-stress boundary conditions at the ends of the crypt to model the sloughing off of cells at the open ends. We also impose the constraint that both ends are fixed at $y=0$, i.e. that

$$
\int_{-1}^{1} \sin \theta \mathrm{d} s=0
$$


If the beam is 'incompressible', by which we mean that it is stiff (i.e. has a large Young's modulus) so that $f(s)=s$, we may solve (1)-(7) for $\theta, F_{x}$ and $F_{y}$. If, however, the beam is 'compressible' so that it can be shortened by the applied force (with a consequent thickening of the beam) then $f(s) \neq s^{1}$. As the beam is a one-dimensional approximation to a twodimensional object the thickening of the layer translates to an increased density $\rho(s)$, and so even an incompressible material with Poisson's ratio $v=1 / 2$ can appear compressible in this approximation. We can determine $f(s)$ from a force balance within the beam, from which we obtain

$$
F_{x} \cos \theta+F_{y} \sin \theta=\lambda_{1}\left(1-\frac{\mathrm{d} f}{\mathrm{~d} s}\right)
$$

in which we measure strain relative to the deformed configuration, and where $\lambda_{1}=E h / k L^{2}$; $E$ is the Young's modulus of the material, and $h$ its thickness. In this case we must also impose a boundary condition on $f(s)$, which we may take from the symmetry of the problem as

$$
f=0 \text { at } s=0 .
$$

Note that as $\lambda_{1} \rightarrow \infty$ in (9) we regain the incompressible model $f(s)=s$. We also note that for an elastic material the bending stiffness $B \sim E h^{3}$ so that $\lambda_{0}=O(1)$ implies that for a thin layer $\lambda_{1} \gg 1$, i.e. that a beam is essentially incompressible. Despite this we will continue to discuss models for which $\lambda_{1}=O(1)$ to allow for the fact that the cells may actively resist bending by altering their internal architecture.

In order to determine when the tissue buckles and initiates budding and fission we must find the values of $\lambda_{0}, F_{0}$, and for the compressible case $\lambda_{1}$, for which the model has a non-flat solution. As we are primarily interested in the initiation of the deformation we simplify the problem by linearising the model about the initial flat state. This gives the linear eigenvalue problem for the deflection $y(x)$

$$
\begin{aligned}
\frac{\mathrm{d}^{4} y}{\mathrm{~d} x^{4}}-\lambda_{0}\left(F_{x} \frac{\mathrm{d}^{2} y}{\mathrm{~d} x^{2}}+\frac{\mathrm{d} F_{x}}{\mathrm{~d} x} \frac{\mathrm{d} y}{\mathrm{~d} x}-y\right) & =0, \\
\frac{\mathrm{d} F_{x}}{\mathrm{~d} x} & =x-f(x),
\end{aligned}
$$

with $f(x)=x$ for an incompressible beam, or with

$$
F_{x}=\lambda_{1}\left(1-\frac{\mathrm{d} f}{\mathrm{~d} x}\right)
$$

and

$$
f=0 \text { at } x=0
$$

for the compressible beam. The linearised boundary conditions are

$$
F_{x}=-F_{0}, \quad \text { and } y=\frac{\mathrm{d} y}{\mathrm{~d} x}=0, \quad \text { at } x= \pm 1 .
$$

1 Often when considering such elastic deformations a mapping $g(X)$ of the initial to the deformed frame is taken so that

$$
(X, 0) \rightarrow\left(\int_{0}^{g(X)} \cos \theta \mathrm{d} s, \int_{-1}^{g(X)} \sin \theta \mathrm{d} s\right)
$$

with $g(0)=0$. However the algebra is significantly simplified by considering instead $f(s)=g^{-1}(s)$, provided that this inverse exists. 


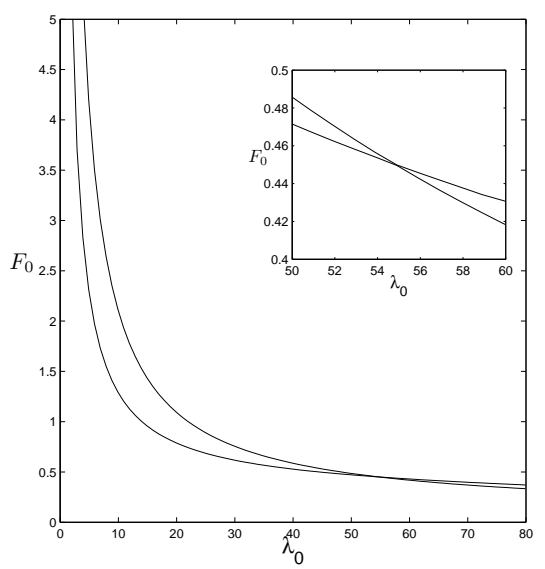

(a)
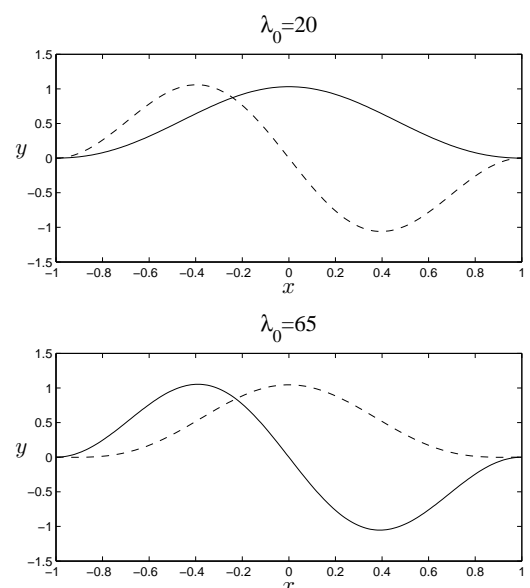

(b)

Fig. 3 (a) A plot of the lowest two eigenvalues of the incompressible model (11),(15) with $F_{x}=-F_{0}$. The inset shows the crossing of the eigenvalues when $\lambda_{0} \approx 55$. (b) A plot of the first two eigenfunctions for $\lambda_{0}=20$ (top) and $\lambda_{0}=65$ (bottom). The solid line corresponds to the lowest eigenfunction.

For the incompressible beam with $f(x)=x$ the solution of (12) and (15) is $F_{x}=-F_{0}$. The numerical solution of the resulting eigenvalue problem (11),(15) is plotted in Figure 3; the solution was obtained using spectral methods in MATLAB as detailed in (Trefethen, 2000) ${ }^{1}$. In Figure 3(a) we plot the lowest two eigenvalues; for $F_{0}$ greater than the lowest eigenvalue there exists a non-zero solution for $y(x)$, which is stable, while the flat solution becomes unstable and so the layer will buckle. As expected, as the bending stiffness tends to infinity, i.e. $\lambda_{0} \rightarrow 0$, the force required to buckle the tissue $F_{0} \rightarrow \infty$. Note that the eigenvalues cross when $\lambda_{0} \approx 55$, as shown in the inset in Figure 3(a). The deflection profiles of the lowest two eigenfunctions for $\lambda_{0}=20$ and $\lambda_{0}=65$ are plotted in Figure $3(\mathrm{~b})$. The solid line indicates the eigenfunction corresponding to the lowest eigenvalue and it can be seen that the qualitative behaviour of the buckled profiles also 'swap' as $\lambda_{0}$ increases above 55 . As the strength of the cellular attachments increases (increasing $\lambda_{0}$ ) so it becomes more energetically favourable for the layer to 'wrinkle' to higher modes, as demonstrated in Appendix A.

For the compressible beam we solve (12)-(15) to find that

$$
F_{x}=-F_{0} \frac{\cosh \left(x / \sqrt{\lambda_{1}}\right)}{\cosh \left(1 / \sqrt{\lambda_{1}}\right)}, \quad f=x+F_{0} \frac{\sinh \left(x / \sqrt{\lambda_{1}}\right)}{\sqrt{\lambda_{1}} \cosh \left(1 / \sqrt{\lambda_{1}}\right)} .
$$

Note that $\left|F_{x}\right|<F_{0}$, i.e. that the force generated within a compressible beam is less than that generated within an incompressible one, and that $F_{x} \rightarrow-F_{0}, f \rightarrow x$ as $\lambda_{1} \rightarrow \infty$. In Figure 4 we plot the numerical solution of the eigenvalue problem obtained by substituting (16) into (11), with $\lambda_{1}=0.09$. The effect of compressibility is, as might have been expected, to increase the force required to buckle the tissue for any particular $\lambda_{0}$. However, the qualitative features of the two models (compressible and incompressible) are the same: $F_{0} \rightarrow \infty$ as $\lambda_{0} \rightarrow$ 0 , and both the eigenvalues and eigenfunctions cross at a particular value of $\lambda_{0}$. Additionally,

1 As (11) now has constant coefficients we could for this particular case find the eigenvalue relationship between $F_{0}$ and $\lambda_{0}$ analytically. However, it is sufficiently complicated that we omit the details, in particular, as this is the only case that will be considered for which this is possible. 


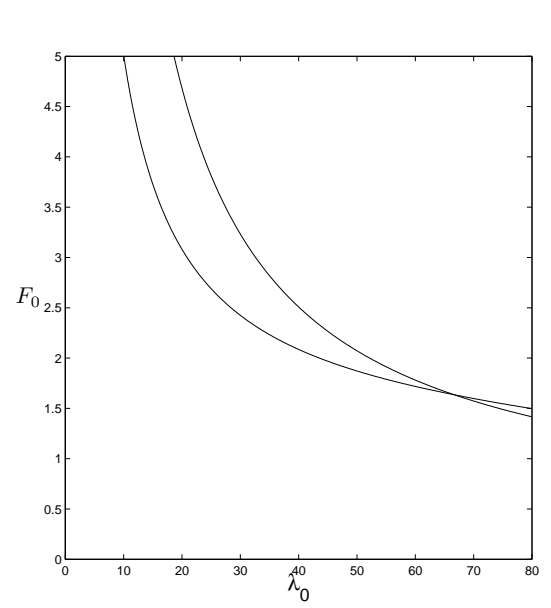

(a)

Fig. 4 (a) A plot of the lowest two eigenvalues of the compressible model (11),(15) with (16) for $\lambda_{1}=0.09$. (b) A plot of the first two eigenfunctions for $\lambda_{0}=20$ (top) and $\lambda_{0}=80$ (bottom). The solid line corresponds to the lowest eigenfunction.

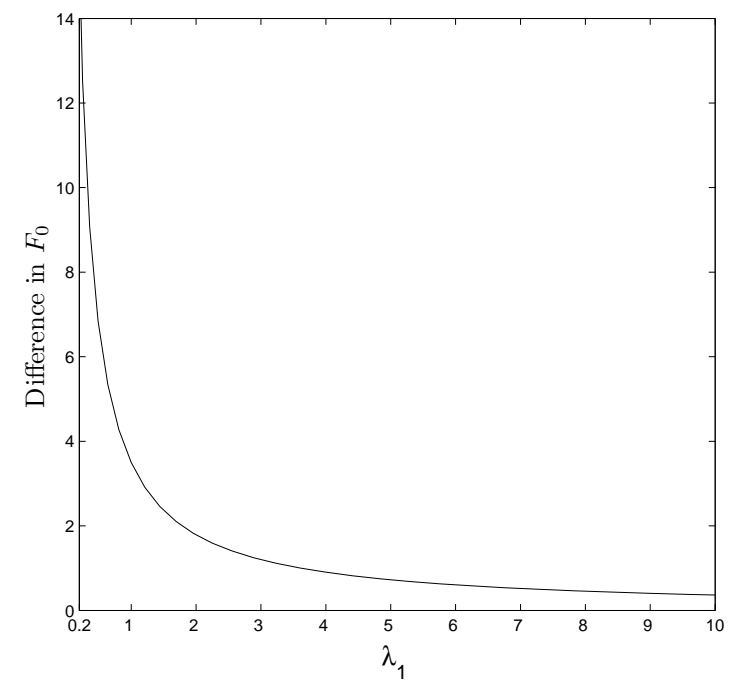

Fig. 5 A plot of the difference in the forces required to buckle an incompressible and compressible epithelial layer for which $\lambda_{0}=1$ as a function of $\lambda_{1}$.

we note that as $\lambda_{1}$ increases the two solutions for the eigenvalues of the incompressible and compressible models quickly become similar away from the origin. In Figure 5, we illustrate this by plotting the difference between the forces required to buckle an incompressible and compressible layer as a function of $\lambda_{1}$ with $\lambda_{0}=1$. 


\section{A model for the crypt}

In the crypt, stress is generated within the epithelial layer not by an externally applied force, but through the growth of the tissue itself. This growth necessitates a more realistic model for the cellular attachments, as including growth in the present description would eventually lead to the unphysical arrangement of all the cells being attached to the origin $x=0$. In fact, as the tissue grows cells move along the crypt attaching and detaching themselves from the basal lamina. This movement, in conjunction with the removal of cells from the top of the crypt, maintains the crypt's normal steady state configuration.

In the light of the results of the previous section we will assume initially that the beam is incompressible (i.e. $\lambda_{1} \gg 1$ ), and will relegate a discussion of the effects of compressibility to Appendix B. We relate $x, t$ to the reference initial configuration by

$$
x=g(X, \tau), \quad t=\tau,
$$

and we take

$$
X=f(x, t), \quad \tau=t
$$

where now the maps $g(X, \tau)$ and $f(x, t)$ are determined by the growth of the tissue. The incompressibility of the beam implies constant density growth with $g(X, \tau)$ satisfying the mass conservation equation

$$
\frac{\mathrm{d}}{\mathrm{d} \tau} \frac{\partial g}{\partial X}=\gamma(x) \frac{\partial g}{\partial X}
$$

with the initial and boundary conditions,

$$
g(0, \tau)=0, \quad \text { and } g(X, 0)=X,
$$

where $\gamma(x)$ is the growth rate of the tissue. We have nondimensionalised time with $\rho / \gamma_{\max }$, where $\rho$ is the density of the tissue and $\gamma_{\max }$ the maximum net proliferation rate (i.e. cell proliferation minus cell death). In practice (19) will be nonlinear as we will know the growth rate $\gamma$ only in the physical frame and not in the reference configuration $X, \tau$. We may recast (19),(20) in terms of $f(x, t)$ as

$$
-\frac{\partial f}{\partial x} \frac{\partial^{2} f}{\partial x \partial t}+\frac{\partial^{2} f}{\partial x^{2}} \frac{\partial f}{\partial t}=\left(\frac{\partial f}{\partial x}\right)^{2} \gamma(x) .
$$

with

$$
f(0, t)=0, \text { and } f(x, 0)=x .
$$

The cells' movement along the lamina, and the consequent relaxation of the horizontal stress generated by the growth, may be modelled by making the component of the tension along the lamina $T_{x}$ viscoelastic. Thus, instead of taking $T_{x}=x-f(x)$ as in (4), we take

$$
\frac{\mathrm{d} T_{x}}{\mathrm{~d} \tau}+\lambda_{\mathrm{T}} T_{x}=\frac{\mathrm{d} x}{\mathrm{~d} \tau}=-\frac{\partial f}{\partial t}\left(\frac{\partial f}{\partial x}\right)^{-1},
$$

with

$$
T_{x}(x, 0)=0,
$$




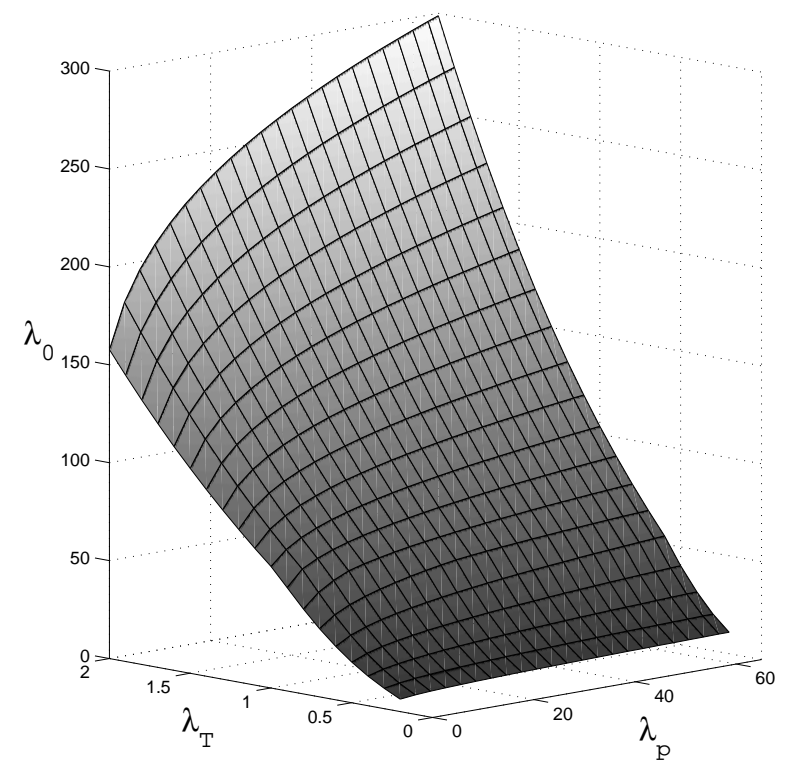

Fig. 6 A plot of the lowest eigenvalue of the system (25)-(27),(31) as a function of $\lambda_{\mathrm{T}}, \lambda_{\mathrm{p}}$. Two sections of this surface with $\lambda_{\mathrm{T}}=0.5$ and $\lambda_{\mathrm{p}}=65$ are also plotted in Figure 7 .

where $\lambda_{\mathrm{T}}$ is the ratio of the growth timescale $\rho / \gamma_{\max }$ to the timescale for the relaxation of the attachments, i.e. the rate at which the cells can move their bonds, and where $f(x, t)$ is determined from the growth as detailed above. Over short times the layer responds elastically, and thus the paradigm problem previously discussed may be interpreted as a guide to its instantaneous behaviour. As the cells stay attached to the lamina at all times we leave $T_{y}$ as purely elastic in response, i.e. $T_{y}=y$.

The model for the growing crypt is thus the linear beam equation

$$
\frac{\mathrm{d}^{4} y}{\mathrm{~d} x^{4}}-\lambda_{0}\left(F_{x} \frac{\mathrm{d}^{2} y}{\mathrm{~d} x^{2}}+\frac{\mathrm{d} F_{x}}{\mathrm{~d} x} \frac{\mathrm{d} y}{\mathrm{~d} x}-y\right)=0
$$

with

$$
\frac{\mathrm{d} F_{x}}{\mathrm{~d} x}=T_{x}
$$

with the tension $T_{x}$ given by (23),(24) and the map $f(x, t)$ by (21),(22). We apply the boundary conditions

$$
F_{x}=0 \text { and } y=\frac{\mathrm{d} y}{\mathrm{~d} x}=0 \quad \text { at } x= \pm 1,
$$

where by taking $F_{x}=0$ at $x= \pm 1$ we model the removal of cells from the top of the crypt.

Unfortunately, this model is fully time dependent. However, we do not need to solve the dynamical problem to determine whether the crypt will deform after a genetic mutation. Instead, we need only determine whether the alteration in the parameters generated by this genetic change destabilises the flat steady state solution. As $t \rightarrow \infty$ we expect the cell velocity 
$-(\partial f / \partial t)(\partial f / \partial x)^{-1}$ to be independent of time, finite and non-zero. This in conjunction with (21) and the boundary conditions (22), gives the long-time solution for $f(x, t)$ as

$$
f(x, t) \sim \alpha(x) e^{-\beta t},
$$

where $\beta$ is a constant and where, from substituting (28) into (21),(22), $\alpha(x)$ satisfies

$$
\left(\frac{\mathrm{d} \alpha}{\mathrm{d} x}\right)^{2}\left(\frac{\gamma}{\beta}-1\right)+\alpha \frac{\mathrm{d}^{2} \alpha}{\mathrm{d} x^{2}}=0, \quad \alpha(0)=0,
$$

which may be integrated to give

$$
\alpha\left(\frac{\mathrm{d} \alpha}{\mathrm{d} x}\right)^{-1}=\int_{0}^{x} \frac{\gamma}{\beta} \mathrm{d} x .
$$

Substituting (28) into (23) we see that in steady state the tension $T_{x}(x)=\alpha \beta\left(\lambda_{\mathrm{T}} \mathrm{d} \alpha / \mathrm{d} x\right)^{-1}$, and so (30) may be expressed as

$$
\frac{\mathrm{d} T_{x}}{\mathrm{~d} x}=\frac{1}{\lambda_{\mathrm{T}}} \gamma(x), \quad T_{x}(0)=0 .
$$

The steady state solution is then given by (25)-(27) with $T_{x}$ given by (31) once the growth rate $\gamma(x)$ is prescribed. (Note that as we are looking for the steady state solution we could have taken any distribution of tension as our initial condition in (24).) In a healthy crypt, cell growth is restricted to the semi-differentiated cells, which occupy roughly the lower three fifths of the crypt (Bach et al., 2000). We model this by taking

$$
\gamma(x)=e^{-\lambda_{\mathrm{p}} x^{6}}
$$

with $\lambda_{\mathrm{p}}=65$, to give a central uniformly proliferating compartment within the crypt that sharply dies back towards zero.

We now look for the solution to the eigenvalue problem (25)-(27),(31), i.e. for the values of $\lambda_{0}, \lambda_{\mathrm{T}}, \lambda_{\mathrm{p}}$ for which a nonzero solution exists. The lowest eigenvalue defines a surface in parameter space marking the boundary between the flat steady state solution and the induction of buckling and crypt budding and fission; below the surface the flat solution is stable and above it this solution is unstable and the layer buckles. We plot this surface in Figure 6 and sections of this surface when $\lambda_{\mathrm{T}}=0.5$ and $\lambda_{\mathrm{p}}=65$ in Figure 7 ; the plots were obtained using spectral methods as before. As discussed in the introduction, an activating mutation in the Wnt cascade is known to increase the size of the proliferative compartment, which will decrease $\lambda_{\mathrm{p}}$; it has also been linked with an increase in net proliferation either through a decrease in apoptosis or cell cycle time, thus decreasing $\lambda_{\mathrm{T}}$ and also with alterations in cell adhesion and migration, which affect both $\lambda_{0}$ and $\lambda_{\mathrm{T}}$. Thus we can see from Figure 6 that the downstream effects of the Wnt cascade can destabilise the flat steady state solution. Note that the amount by which we must change any parameter value before buckling the layer is dependent on the values of the other two parameters. Indeed, as can be seen from Figure $7(a)$, even with all the cells in the crypt proliferating (i.e. $\lambda_{p}=0$ ), $\lambda_{0}$ still has to be greater than some critical value before buckling can occur. Thus we must consider the combined effect of the changes in each of the parameters $\lambda_{0}, \lambda_{\mathrm{p}}, \lambda_{\mathrm{T}}$ as a result of any mutational change.

In Figure 6(b), we also plot the second eigenvalue with $\lambda_{\mathrm{p}}=65$ and it is interesting to note that the crossing of the eigenvalues (and eigenfunctions) noted for the paradigm problem is again a feature of the solution. 


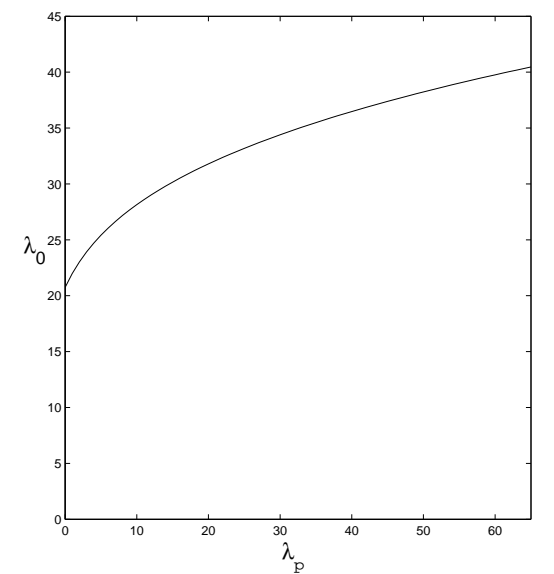

(a)

Fig. 7 (a) The lowest eigenvalue of the system (25)-(27),(31) as a function of $\lambda_{\mathrm{p}}$ with $\lambda_{\mathrm{T}}=0.5$. (b) The solid line corresponds to the lowest two eigenvalues of the system (25)-(27),(31) as a function of $\lambda_{\mathrm{T}}$ with $\lambda_{\mathrm{p}}=65$. The lowest eigenvalue corresponds to a section of Figure 6 . The dashed line corresponds to the lowest two eigenvalues with $\lambda_{p}=65$ when compressibility is included in the model $\left(\lambda_{1}=1\right)$.

\section{Conclusions}

The model we have presented in Section 3 shows how crypt budding and fission could be biomechanically initiated as a result of activating mutations in the Wnt cascade. The three downstream effects of this mutation that effect crypt shape are: stopping the cells differentiating and therefore increasing the proliferative compartment, increasing the net proliferation rate either by decreasing apoptosis or increasing the cellular proliferation, and changes in cell adhesion and motility. The effect of these changes on the model is to alter the values of the parameters $\lambda_{\mathrm{p}}$, which quantifies the size of the proliferative compartment, $\lambda_{\mathrm{T}}$, which quantifies the competition between the rates of cell growth and cell movement, and $\lambda_{0}$, which quantifies the relative importance of spring strength against bending stiffness. We have shown that any combination of changes that move the parameters across the surface of eigenvalues plotted in Figure 6(a) will buckle the tissue. For example, we have shown that increasing the net proliferation, or the time taken for a cell to 'move' its attachments, i.e. decreasing $\lambda_{\mathrm{T}}$, can initiate buckling independently of the the other parameters. Equally, it can be seen that enlarging the proliferative compartment, provided $\lambda_{0}$ is large enough, will also initiate buckling. We suggest that this buckling could initiate crypt budding and fission.

In Appendix B we show that compressibility of the layer further destabilises the crypt shape, although the results of the model are qualitatively similar to those obtained in Section 3. However, we expect this to be a higher order effect for, as was discussed in Section 2, unless a cell can significantly increase its bending stiffness the layer should be considered to be stiff and essentially incompressible.

Finally, we remark that this modelling framework can be extended to consider more realistic three-dimensional geometries. Also we note that in reality as the epithelial layer deforms it draws the lamina propria up with it, leading to a permanent deformation of this tissue. Thus in order to describe the progression of the budding and fission events past their 
initiation it will be necessary to model this interaction between the epithelium and the lamina propria.

Acknowledgements The authors thank Prof. P.K. Maini, Dr H.M. Byrne, and Dr I.M.M. van Leeuwen for useful discussions, and gratefully acknowledge the support provided by the funders of the Integrative Biology project: the EPSRC (GR/S720231/01) and IBM.

\section{A The buckling of a beam with $\lambda_{0} \gg 1$}

We again consider the simple paradigm problem of Section 2 in the limit $\lambda_{1} \rightarrow \infty$ so that the beam is incompressible. The eigenvalue problem is in this case (11) with $F_{x}=-F_{0}$, with the clamped boundary conditions (15). When $\lambda_{0} \ll 1$ the first two terms in equation (11) balance and the solution is no longer influenced by the underlying mucosa; the problem reduces to a classical Euler strut problem. However, as $\lambda_{0} \rightarrow \infty$ so the attachment becomes stronger as compared with the bending stiffness of the beam. In order to understand how the beam behaves in this limit we look for a WKB expansion (Bender and Orszag, 1999) of the solution to (11),(15), which we write for convenience as

$$
\begin{aligned}
& \frac{\mathrm{d}^{4} y}{\mathrm{~d} x^{4}}+\alpha k^{2} \frac{\mathrm{d}^{2} y}{\mathrm{~d} x^{2}}+k^{4} y=0, \\
& y=0=\frac{\mathrm{d} y}{\mathrm{~d} x} \text { at } x= \pm 1,
\end{aligned}
$$

where $\alpha k^{2}=F_{0} \lambda_{0}, k^{4}=\lambda_{0}$, and $k \gg 1$. Substituting the WKB Ansatz

$$
y \sim A(x) e^{i k \phi(x)}, A(x) \sim A_{0}(x)+\frac{1}{k} A_{1}(x)+O\left(\frac{1}{k^{2}}\right),
$$

into (33) and comparing powers of $k$ up to $O\left(k^{3}\right)$ we find that for a non-zero solution satisfying the boundary conditions (34) to exist we require

$$
\alpha=2+\varepsilon, \text { and } \phi(x)=\phi_{0} \sim 1+O(\sqrt{\varepsilon}),
$$

where $\varepsilon \ll 1$. At $O\left(k^{2}\right)$ we find that

$$
\frac{\mathrm{d}^{2} A_{0}(x)}{\mathrm{d} x^{2}}=-\frac{1}{4} \varepsilon k^{2} A_{0}(x) .
$$

In order to satisfy the boundary conditions (34) $\varepsilon=\pi^{2} / k^{2}$, and so as $\lambda_{0} \rightarrow \infty$, we see from (36) and (33) that the eigenvalue relation between $F_{0}$ and $\lambda_{0}$ is given by

$$
\alpha=2+\frac{\pi^{2}}{k^{2}} \Longrightarrow F_{0}=\frac{2}{\sqrt{\lambda_{0}}}+\frac{\pi^{2}}{\lambda_{0}} .
$$

and

$$
y \sim A_{0} \cos (\pi x / 2) e^{i \lambda_{0}^{1 / 4} x}+\ldots,
$$

Thus we see that in the limit $\lambda_{0} \rightarrow \infty$ the layer 'buckles' into a wrinkled state with an envelope looking like $\cos (\pi x / 2)$, with the force required to buckle the layer given by (38).

\section{B Including compressibility in the model of Section 3}

If we consider the layer of Section 3 to be compressible then we must account for its deformation as it grows, as $f(x, t)$ cannot be determined from conservation of mass alone. The standard approach in this case is to decompose the map $g(X, \tau)$, considering that the tissue first grows under the constant density assumption to a zero stress configuration $g_{0}(X, \tau)$, and is then subsequently deformed to its final configuration $g(X, \tau)$; see 
for example (Ambrosi and Mollica, 2002; Lubarda and Hoger, 2002). The elastic deformation of the material is given by an equation analogous to (9) but with the strain calculated from the displacement from the zero stress configuration, so that (after linearisation)

$$
F_{x}=\lambda_{1}\left(1-\frac{\partial g_{0}}{\partial X} \frac{\partial f}{\partial x}\right)
$$

with $g_{0}(X, \tau)$ determined from (19),(20) with $g$ replaced by $g_{0}$. The model for a compressible crypt is thus (25)-(27),(23),(24), with (19),(20),(40),(22) in which $g$ is replaced by $g_{0}$.

To determine the stability of the steady solution of this model, we assume that both the cell velocity profile $\mathrm{d} x / \mathrm{d} \tau$, and $F_{x}$ tend to a finite limit as $t, \tau \rightarrow \infty$, which gives $f(x, t) \sim \alpha(x) e^{-\beta t}$ for $t$ large, and from (40) that

$$
\frac{\partial f}{\partial x} \frac{\partial g_{0}}{\partial X} \rightarrow H(x) \text { as } \tau, t \rightarrow \infty
$$

giving $\partial g_{0}(X, \tau) / \partial X=\partial g_{0}(f(x, t), t) / \partial X \sim H(x) e^{\beta t}(\mathrm{~d} \alpha / \mathrm{d} x)^{-1}$ for $\tau, t$ large. Substituting these expressions for $f$ and $g$ into (19),(23) (and remembering that $\mathrm{d} / \mathrm{d} \tau=\partial / \partial t+\mathrm{d} x / \mathrm{d} t \cdot \partial / \partial x$ ) gives

$$
\frac{\mathrm{d} H T_{x}}{\mathrm{~d} x}=\frac{1}{\lambda_{\mathrm{T}}} \gamma H
$$

where $H$ is given by (40),(26), i.e. by

$$
\begin{aligned}
F_{x} & =\lambda_{1}(1-H), \\
\frac{\mathrm{d} F_{x}}{\mathrm{~d} x} & =T_{x},
\end{aligned}
$$

with the boundary conditions $H(1)=1, T_{x}(0)=0$. Note that as $\lambda_{1} \rightarrow \infty, H \rightarrow 1$ and we regain the incompressible model.

Equations (42)-(44),(25) and the boundary conditions (27) and $H(1)=0$ and $T_{x}(0)=0$ define the eigenvalue problem for the steady state of the compressible beam. The surface defining the boundary between the flat and buckled states may be obtained as in Section 3, and is found to be qualitatively similar to that displayed in Figure 6(a). The compressibility of the layer does, however, lead to a surprising quantitative difference; a compressible layer is found to be easier to buckle that an incompressible one. This is easiest to see when comparing sections of the surface of eigenvalues, and in Figure 6(b) we plot as dotted lines the lowest two eigenvalues for the case $\lambda_{\mathrm{p}}=65, \lambda_{1}=1$. It can be seen from Figure 6(b) that the compressible eigenvalues lie below those found for the incompressible layer. That this should be the case, in contrast to the result of Section 2, where it was found that for a simple tethered beam compressibility made the layer harder to buckle, can be shown by taking $\lambda_{1}$ large but finite and expanding $F_{x}, T_{x}, H$ as power series in $1 / \lambda_{1}$, i.e.

$$
\begin{aligned}
& F_{x}(x) \sim F_{x 0}(x)+\frac{1}{\lambda_{1}} F_{x 1}(x)+O\left(\frac{1}{\lambda_{1}^{2}}\right), \\
& T_{x}(x) \sim T_{x 0}(x)+\frac{1}{\lambda_{1}} T_{x 1}(x)+O\left(\frac{1}{\lambda_{1}^{2}}\right), \\
& H(x) \sim H_{0}(x)+\frac{1}{\lambda_{1}} H_{1}(x)+O\left(\frac{1}{\lambda_{1}^{2}}\right) .
\end{aligned}
$$

Substituting (45)-(47) into (42)-(44) and applying the boundary conditions $H(1)=1, T_{x}(0)=0$ and (27) we find that at lowest order, as expected, $F_{x 0}, T_{x 0}, H_{0}$ are given by the solution to the incompressible model, i.e.

$$
\begin{aligned}
H_{0} & =1, \\
T_{x 0} & =\int_{0}^{x} \frac{1}{\lambda_{\mathrm{T}}} \gamma \mathrm{d} x, \\
F_{x 0} & =-\int_{x}^{1} T_{x 0} \mathrm{~d} x .
\end{aligned}
$$

Looking at terms of $O\left(1 / \lambda_{1}\right)$ we then find that

$$
F_{x 1}=-\int_{x}^{1} \int_{0}^{v} T_{x 0}^{2} \mathrm{~d} x \mathrm{~d} v,
$$


and thus that the force generated within the compressible layer is greater than that generated in the incompressible one. This may be explained as follows. The compression in the layer is greatest in the centre, so that in the compressible case more material is squeezed in there. As the cells move along the crypt the compression is reduced, so that the material expands giving it a greater velocity and therefore generating a greater tension in the viscoelastic tethers. However, we note, as discussed in Section 2, that we expect for any thin layer that $\lambda_{1} \gg 1$, and so this effect will be very small in comparison to that of changes in $\lambda_{\mathrm{p}}, \lambda_{\mathrm{T}}$ and $\lambda_{0}$.

\section{References}

Ambrosi, D., Mollica, F., 2002. On the mechanics of a growing tumor. Int. J. Eng. Sci. 40, 1297-1316

Araki, K., Ogata, T., Kobayashi, M., Yatani, R., 1995. A morphological study on the histogenesis of human colorectal hyperplastic polyps. Gastroenterology 109, 1468-1474

Bach, S.P., Renehan, A.G., Potten, C.S., 2000. Stem cells: the intestinal stem cell as a paradigm. Carcinogenesis $21,469-476$

Bender, C.M., Orszag, S.A., 1999. Advanced Mathematical Methods for Scientists and Engineers. Springer, New York

Brittan, M., Wright, N.A., 2004. Stem cell in gastrointestinal structure and neoplastic development. Gut 53, 899-910

Brush, D.O., Almroth, B.O., 1975. Buckling of Bars, Plates, and Shells. McGraw-Hill, New York

Cummings, F.W., 1990. A model of morphogenetic pattern formation. J. Theor. Biol. 144, 547-566

Davidson, L.A., Koehl, M.A.R., Keller, R., Oster, G.F., 1995. How do sea urchins invaginate? Using biomechanics to distinguish between mechanisms of primary invagination. Development 121, 2005-2018

Drasdo, D., Loeffler, M., 2001. Individual-based models to growth and folding in one-layered tissues: Intestinal crypts and early development. Nonlinear Analysis 47, 245-256

Fearon, E.R., Vogelstein, B., 1990. A genetic model for colorectal tumorigenesis. Cell 61, 759-767

Fodde, R., 2003. The multiple functions of tumour suppressors: it's all in APC. Nature Cell Biology 5, 190192

Greaves, L.C., Preston, S.L., Tadrous, P.J., Taylor, R.W., Barron, M.J., Oukrif, D., Leedham, S.J., Deheragoda, M., Sasieni, P., Novelli, M.R., Jankowski, J.A.Z., Turnbull, D.M., Wright, N.A., McDonald, S.A.C., 2006. Mitochondrial DNA mutations are established in human colonic stem cells, and mutated clones expand by crypt fission. PNAS 103, 714-719

Hunt, G.W., Bolt, H.M., Thompson, J.M.T., 1989. Structural localization phenomena and the dynamical phase-space analogy. Proc. R. Soc. Lond. A 425, 245-267

Keller, R., Davidson, L.A., Shook, D.R., 2003. How we are shaped: The biomechanics of gastrulation. Differentiation 71, 171-205

Lubarda, V.A., Hoger, A., 2002. On the mechanics of solids with a growing mass. Int. J. Solids Struct. 39, 4627-4664

Marshman, E., Booth, C., Potten, C.S., 2002. The intestinal epithelial stem cell. BioEssays 24, 91-98

Nelson, W.J., Nusse, R., 2004. Convergence of Wnt, $\beta$-catenin, and cadherin pathways. Science 303, $1483-$ 1487

Odell, G.M., Oster, G., Alberch, P., Burnside, B., 1981. The mechanical basis of morphogenesis. I. Epithelial folding and invagination. Developmental Biology 85, 446-462

Preston, S.L., Wong, W-M., Chan, A. O-O., Poulsom, R., Jeffery, R., Goodlad, R.A., Mandir, N., Elia, G., Novelli, M., Bodmer, W.F., Tomlinson, I.P., Wright, N.A., 2003. Bottom-up histogenesis of colorectal adenomas: origin in the monocryptal adenoma and initial expansion by crypt fission. Cancer Research 63, 3819-3825

Renehan, A.G., O’Dwyer, S.T., Haboubi, N.J., Potten, C.S., 2002. Early cellular events in colorectal carcinogenesis. Colorectal disease 4, 76-89

Reya, T., Clevers, H., 2005. Wnt signalling in stem cells and cancer. Nature 434, 843-850

Roncucci, L., Pedroni, M., Vaccina, F., Benatti, P., Marzona, L., DePol, A., 2000. Aberrant crypt foci in colorectal carcinogenesis. Cell and crypt dynamics. Cell Prolif. 33, 1-18

Samsom, O.J., Reed, K.R., Hayes, A.J., Ireland, H., Brinkmann, H., Newton, I.P., Batlle, E., Simon-Assmann, P., Clevers, H., Nathke, I.S., Clarke, A.R., Winton, D.J., 2004. Loss of APC in vivo immediately perturbs Wnt signalling, differentiation and migration. Genes and Development 18, 1385-1390

Shih, L-M, Wang, T-L, Traverso, G., Romans, K., Hamilton, S.R., Ben-Sasson, S., Kinzler, K.W., Vogelstein, B., 2001. Top-down morphogenesis of colorectal tumours. PNAS 98, 2640-2645

Timoshenko, S., Lessells, J.M., 1928. Applied elasticity. Constable and Co., London

Trefethen, L.N., 2000. Spectral Methods in Matlab. SIAM, Philadelphia, PA 
Wong, W-M., Mandir, N., Goodlad, R.A., Wong, B.C.Y., Garcia, S.B., Lam, S-K., Wright, N.A., 2002. Histogenesis of human colorectal adenomas and hyperplastic polyps: the role of cell proliferation and crypt fission. Gut 50, 212-217 